\title{
New Calibration Technique for Microwave Moisture Sensors
}

\author{
Samir Trabelsi, Senior Member, IEEE, Andrzej W. Kraszewski, Fellow, IEEE, and Stuart O. Nelson, Fellow, IEEE
}

\begin{abstract}
A new calibration technique was developed for implementation with microwave moisture sensors. The calibration permittivity function used for this purpose allows computation of moisture content in granular materials with significant differences in shape, dimensions, and composition, independent of bulk density and with temperature compensation. A three dimensional (3-D) representation is used to plot the calibration permittivity function as it depends on temperature and moisture content in wheat and corn. For each material, data points form a plane surface. These planes have nearly the same coefficients, which can be utilized for the development of a "universal" calibration method for moisture sensing in natural and manufactured granular materials. Foundations of the method are discussed based on results obtained for wheat and corn over a wide temperature range and at moisture contents of practical interest.
\end{abstract}

Index Terms-Corn (maize), density independence, dielectric properties, granular materials, material independence, microwave moisture sensor, universal calibration, wheat.

\section{INTRODUCTION}

D IELECTRIC methods are increasingly being used for the development of reliable sensors for on-line determination of physical properties of materials such as bulk density and moisture content without disturbing the process in progress [1]-[6]. Unlike most conventional techniques, these methods provide real-time information nondestructively and, in some instances, without requiring any contact with the material. The essence of microwave dielectric methods for moisture sensing is based on the coupling between the electromagnetic energy of the incident wave and the material under consideration.

It is well known that water strongly affects the material relative complex permittivity, $\epsilon=\epsilon^{\prime}-j \epsilon^{\prime \prime}$, where $\epsilon^{\prime}$ is the dielectric constant and $\epsilon^{\prime \prime}$ is the dielectric loss factor. However, it is not the only property influencing the complex permittivity. It is also dependent on frequency, temperature, bulk density, and composition [5]. Hence, accurate determination of moisture content in a given material from measurement of its dielectric properties relies on development of calibration methods that eliminate, or account for, effects of variables other than moisture content. Commonly, this is reflected in the calibration equation by correction and/or compensation factors related to the different variables. Also, because of differences in structural geometry and composition, an individual calibration equation is required for each material. From sensor design and implementation stand-

Manuscript received May 4, 2000; revised February 27, 2001.

The authors are with the U.S. Department of Agriculture, Agricultural Research Service, Richard B. Russell Agricultural Research Center, Athens, GA 30604-5677 USA (e-mail: strabelsi@qaru.ars.usda.gov).

Publisher Item Identifier S 0018-9456(01)06015-6. points, this will translate into the integration of several different sensing devices (bulk density and temperature, for example) and multiple computing steps that increase the overall error. All of these aspects complicate the design, implementation, and maintenance of a moisture sensor dedicated to routine on-line measurements.

A better approach, which is more viable technically and economically, consists of minimizing the number of correction and compensation factors in the calibration equation by defining a calibration function that is less sensitive to variation in variables other than moisture content. Ideally, the effects of these variables are eliminated instead of compensated, and the same calibration equation covers a wide range of materials.

In this study, a permittivity-based calibration function $\psi$ is used to investigate foundations of a calibration technique for a "universal" type microwave moisture meter. This calibration function is both density-independent and material-independent [7]. Therefore, at a given frequency, the only remaining variable to be accounted for is temperature. To validate this approach, two granular materials, corn and wheat, with pronounced differences in kernel geometry, dimensions, and, composition are considered. A three-dimensional (3-D) analysis was used to generate moisture calibration equations with temperature compensation. Although, wheat and corn present significant differences, their moisture calibration equations are nearly the same, with a standard error of calibration (SEC) of less than $1 \%$ moisture content, wet basis. This finding, with previously reported results, demonstrates the potential of this new calibration technique for the development of a "universal" microwave moisture sensor for granular and particulate materials [5]-[9].

\section{Physical Properties and CALIBration PERMITTIVITY FUNCTION}

Here, the term physical properties of granular materials, refers to the bulk density $(\rho)$, moisture content $(M)$, temperature $(T)$, and the geometry and composition of the granular materials. The bulk density is determined by weighing a given volume $v$ of material as follows

$$
\rho=\frac{m_{w}+m_{d}}{v}
$$

where $m_{w}$ is the mass of water and $m_{d}$ is the mass of dry matter of the sample. The wet-basis moisture content, in percent, is defined as

$$
M=\frac{m_{w}}{m_{w}+m_{d}} \times 100 .
$$

Moisture content is conventionally determined by oven-drying techniques under well defined conditions [10]. Temperature is 
TABLE I

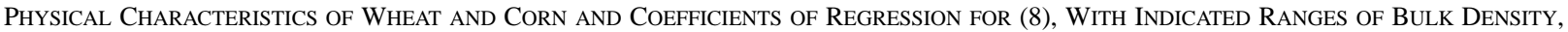
MOISTURE CONTENT, AND TEMPERATURE

\begin{tabular}{ccccccccccc}
\hline Material & $d(\mathrm{~cm})$ & Kernel shape & Kernel length $(\mathrm{mm})$ & $\rho\left(\mathrm{g} / \mathrm{cm}^{3}\right)$ & $M(\%)$ & $T(\mathrm{~K})$ & $a$ & $b$ & $c$ & $r^{2}$ \\
\hline Wheat & 10.4 & Ellipsoidal & $5-7$ & $0.72-0.88$ & $10.6-19.2$ & $272-318$ & 0.0022 & 0.0174 & 0.620 & 0.988 \\
Corn & 5.2 & Oblate & $12-13$ & $0.69-0.83$ & $9.0-19.2$ & $277-308$ & 0.0023 & 0.0166 & 0.593 & 0.968 \\
\hline
\end{tabular}

usually measured with thermometers or thermocouple or thermistor devices.

All three variables, $\rho, M$, and $T$, have similar effects on the relative complex permittivity [6], [11]. In cereal grains, the permittivity increases when $\rho, M$, or $T$ increase. The remaining two physical properties, structural geometry and composition, are known to affect the microwave scattering and absorption properties of a given material. However, they have not been investigated quantitatively. In general, use of the relative complex permittivity for moisture content determination in a given material is conditioned by compensation for effects of all other variables.

Recently, a permittivity function that is independent of the bulk density, kernel geometry and dimensions, and composition was defined in terms of the dielectric properties, based on the principle of energy distribution between stored and dissipated electric energy and the complex-plane representation of the relative complex permittivity normalized to bulk density [5]-[7]. This permittivity function is expressed analytically as

$$
\psi=\sqrt{\frac{\epsilon^{\prime \prime}}{\epsilon^{\prime}\left(a_{f} \epsilon^{\prime}-\epsilon^{\prime \prime}\right)}}
$$

where $a_{f}$ is a frequency-dependent coefficient. The density- independent and material-independent character of $\psi$ has been shown for four granular materials, i.e., wheat, Triticum aestivum L., corn (maize), Zea mays L., oats, Avena sativa L., and rapeseed, Brassica napus L., over wide ranges of frequency, temperature, bulk density, and moisture content [5]-[8]. At a given frequency, $\psi$ increases linearly with moisture content and temperature [9]. Therefore, when $\psi$ is used as a calibration function for moisture prediction from relative complex permittivity measurement, the only variable that needs compensation is temperature. The main advantage of $\psi$ is its exclusive expression in terms of the intrinsic electrical properties of the material, providing more flexibility in the choice of the appropriate measurement technique for a given application.

\section{Calibration Procedures}

Calibration of a measuring system for routine on-line use is the most critical phase in the implementation of such a system because it reflects the validity of the measurement technique and repeatability of the measurements. Because of the dependence of $\psi$ on the relative complex permittivity, the performance of such a calibration function is mainly related to the measurement accuracy and repeatability for determining the dielectric constant $\epsilon^{\prime}$ and the loss factor $\epsilon^{\prime \prime}$. In the following, a detailed description of the different steps for the generation of moisture calibration equations with temperature compensation is given for wheat and corn at a single microwave frequency. These steps include (A) measurement of the two components of the relative complex permittivity, (B) determination of the coefficient $a_{f}$ from the complex-plane representation of the relative complex permittivity divided by bulk density, (C) correlation of the calibration permittivity function $\psi$ with moisture content and temperature, and, finally, (D) the establishment of moisture calibration equations with temperature compensation.

\section{A. Measurement of the Two Components of the Relative Complex Permittivity}

Different measurement techniques can be used to determine the dielectric constant $\epsilon^{\prime}$ and the loss factor $\epsilon^{\prime \prime}$ of dielectric materials [12], [13]. Among these techniques, the most commonly used are transmission line, impedance, and resonant cavity measurements. The choice of the most suitable measurement technique is conditioned by the configuration of a given line, where the product to be measured is being processed, and error tolerance. Very often, free-space techniques (reflection and transmission) are selected because these can be easily adapted to a given configuration and provide accurate measurement of the two components of the relative complex permittivity remotely and nondestructively. In this study, $\epsilon^{\prime}$ and $\epsilon^{\prime \prime}$ of wheat and corn were measured by using a free-space transmission technique [9], [14].

A layer of material of thickness $d$ of known bulk density, moisture content, and temperature (Table I) was placed between two horn antennas (transmitter and receiver), and the attenuation $A$ and phase shift $\phi$ were measured for each sample. For a plane wave propagating through a low-loss material, $\epsilon^{\prime}$ and $\epsilon^{\prime \prime}$ are calculated from the following equations:

$$
\begin{aligned}
\epsilon^{\prime} & =\left(\frac{\beta}{\beta_{0}}\right)^{2} \\
\epsilon^{\prime \prime} & =\frac{2 \alpha \beta}{\beta_{0}^{2}}
\end{aligned}
$$

where

$$
\begin{array}{ll}
\alpha=A / d & \text { attenuation constant; } \\
\beta=\phi / d+\beta_{0} & \text { phase constant; } \\
\beta_{0}=2 \pi / \lambda_{0} & \text { phase constant for free-space wavelength } \lambda_{0} .
\end{array}
$$

\section{B. Determination of Frequency-Dependent Coefficient $a_{f}$}

Figs. 1 and 2 show the complex-plane representation of the relative complex permittivity divided by bulk density for wheat 


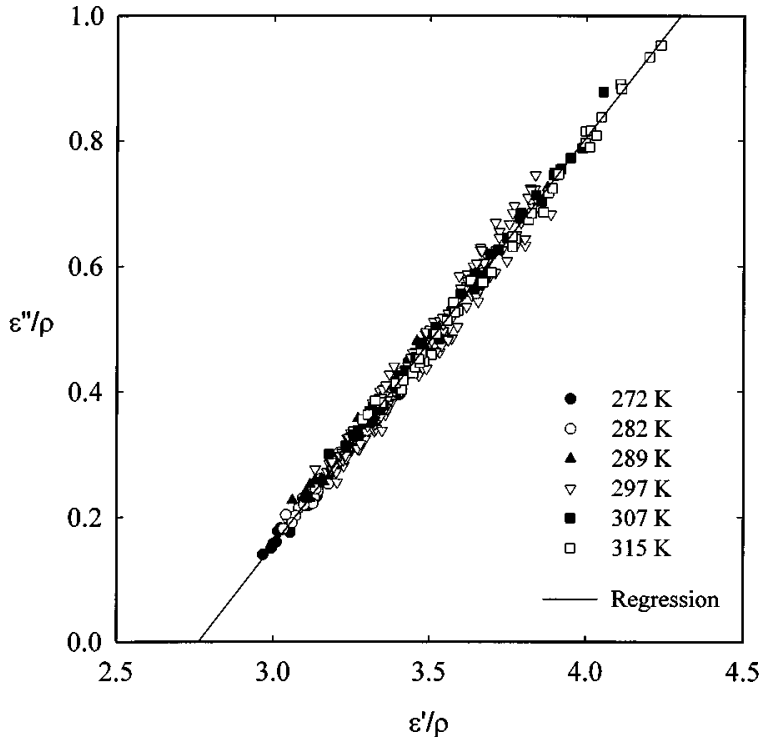

Fig. 1. Argand diagram of the relative complex permittivity divided by bulk density for hard red winter wheat at $14.2 \mathrm{GHz}$ and indicated temperatures.

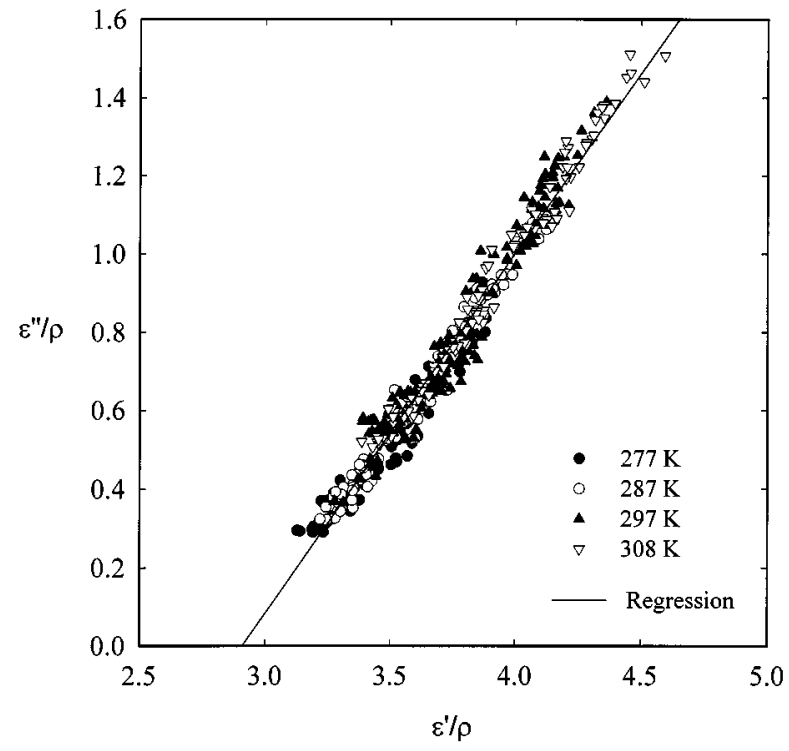

Fig. 2. Argand diagram of the relative complex permittivity divided by bulk density for yellow-dent field corn (maize) at $14.2 \mathrm{GHz}$ and indicated temperatures.

and corn, respectively, at $14.2 \mathrm{GHz}$ and indicated temperatures. Data corresponding to samples of different temperatures fall along the same straight line showing the interchangeability of moisture content and temperature [8]. The slope of the straight line is the coefficient $a_{f}$ which is dependent on the frequency alone. Measurements at several frequencies between $11.3 \mathrm{GHz}$ and $18.0 \mathrm{GHz}$ show that $a_{f}$ for wheat and corn, respectively, is related to the frequency as follows [8], [9]

$$
\begin{array}{ll}
a_{f}=0.0184 F+0.3826 & r^{2}=0.993 \\
a_{f}=0.0571 F+0.1106 & r^{2}=0.989
\end{array}
$$

where $F$ is the frequency in $\mathrm{GHz}$ and $r^{2}$ is the coefficient of determination for the linear regression. At $14.2 \mathrm{GHz}, a_{f}$ is 0.64 for

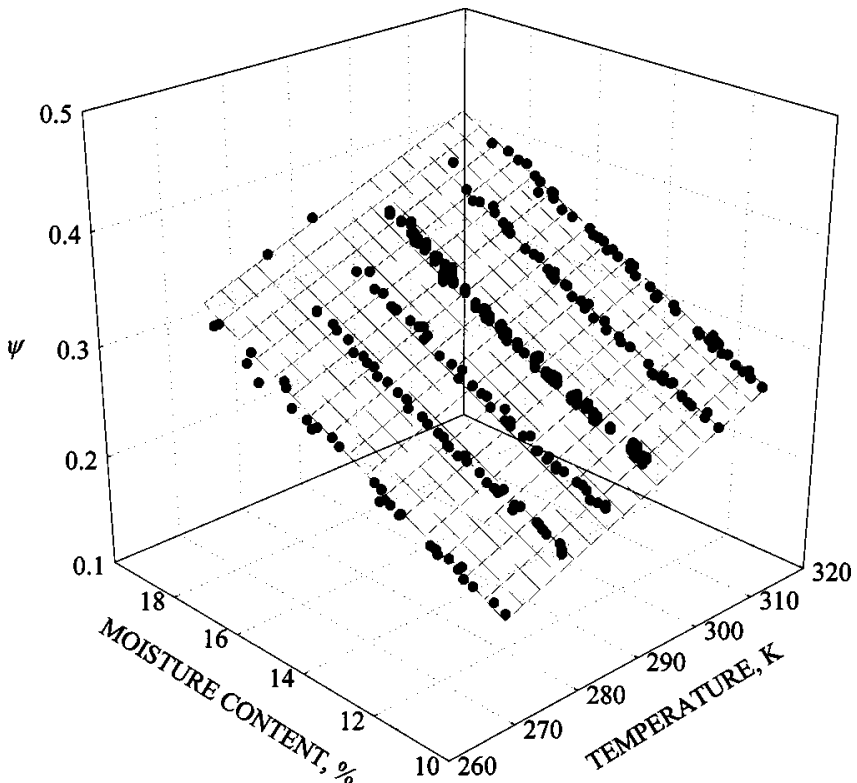

Fig. 3. Moisture and temperature dependence of density-independent function $\psi$ at $14.2 \mathrm{GHz}$ for hard red winter wheat.

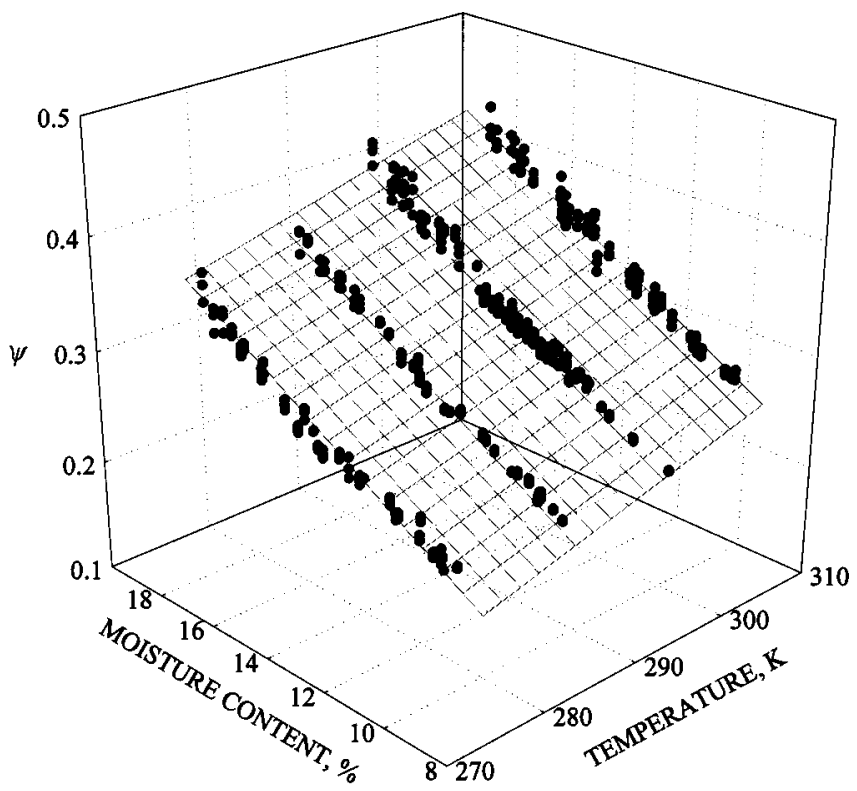

Fig. 4. Moisture and temperature dependence of density-independent function $\psi$ at $14.2 \mathrm{GHz}$ for yellow-dent field corn (maize).

wheat and 0.92 for corn. For $a_{f}$ determination, measurements at one frequency and one temperature are sufficient, provided that the desired ranges of moisture contents and bulk densities are represented.

\section{Correlation of the Calibration Permittivity Function $\psi$ with Moisture Content and Temperature}

After obtaining $\epsilon^{\prime}$ and $\epsilon^{\prime \prime}$ from step A, (4) and (5), and $a_{f}$ from step B, (6) or (7), the calibration permittivity function $\psi$ is computed for each sample by using (3). Figs. 3 and 4 show the variation of $\psi$ as a function of temperature and moisture content at $14.2 \mathrm{GHz}$ for wheat and corn, respectively. These 3-D representations reveal that data points obtained for each material lie 
in the same plane, forming a network of nearly parallel straight lines. The equation of the plane is of the form

$$
\psi=a T+b M+c .
$$

For each material, a regression analysis provides the coefficients $a, b$, and $c$ for (8) as listed in Table I for indicated ranges of bulk density, moisture content, and temperature. Although wheat and corn kernels have pronounced differences in shape, dimensions, and composition, the coefficients of the fitting equations are nearly the same (Table I), implying that a single calibration equation can be used to predict moisture content in wheat and corn from measurement of their dielectric properties at microwave frequencies.

\section{Moisture Calibration Equations with Temperature Compensation}

The moisture calibration equation is determined by solving equation (8) for $M$

$$
M=\frac{\psi-a T-c}{b}
$$

where $a, b$, and $c$ are constants determined in step C, from (8), and $T$ is the temperature in Kelvin. These constants are given in Table I for wheat and corn, respectively. Given the dependence of $M$ on $\psi$ and $T$, errors in moisture prediction from (9) are mainly related to errors in dielectric properties and temperature measurements. Effectiveness of (9) in predicting moisture content in wheat and corn can be evaluated by calculating the standard error of calibration (SEC) which is defined as

$$
\mathrm{SEC}=\sqrt{\frac{1}{n-p-1} \sum_{i=1}^{n}\left(\Delta m_{i}\right)^{2}}
$$

where

$n$ number of samples;

$p$ number of variables in the regression equation with which the calibration is performed;

$\Delta m_{i}$ difference between the predicted value and that determined by a standard method for the $i$ th sample.

The SEC was $0.8 \%$ and $0.9 \%$ moisture content, wet basis, for wheat and corn, respectively.

Once the different calibration steps are accomplished, (9) can be programmed in a measuring system for routine on-line measurement of moisture content in granular and particulate materials.

\section{CONCLUSION}

In the last two decades, microwave moisture sensors have been used successfully to monitor and/or control moisture content in different natural and manufactured granular and particulate materials. The main obstacles for their implementation are cost and, more importantly, the need for customized design and calibration for each particular application for a given material. It has been shown that a single permittivity-based calibration function for moisture sensing in two very different cereal grains, wheat and corn (maize), provides results with standard errors of less than $1 \%$ moisture content with measurements at a single microwave frequency, independent of bulk density variation and compensated for temperature. By defining a calibration permittivity function that is both density- and material-independent, there is an enhanced opportunity for the development of a cost-effective, "universal" microwave moisture meter. Moreover, the availability of new microwave components of reduced size at affordable price will make dielectric-based moisture sensing methods even more viable in the future.

\section{REFERENCES}

[1] A. Kraszewski, Microwave Aquametry-Electromagnetic Wave Interaction with Water-Containing Materials. New York: IEEE Press, 1996.

[2] H. Baltes, W. Göpel, and J. Hesse, Eds., Sensors Update, K. Kupfer, A. Kraszewski, and R. Knöchel, Eds. Weinheim, Germany: Wiley-VCH, 2000, vol. 7, RF and Microwave Sensing of Moist Materials, Food and other Dielectrics.

[3] M. Kent and E. Kress-Rogers, "Microwave moisture and density measurements in particulate solids," Trans. Inst. Meas. Contr., vol. 8, pp. $161-168,1986$

[4] F. Menke and R. Knöchel, "New density-independent moisture measurement methods using frequency-swept microwave transmission," in MTT-S Symp. Dig., vol. 3, 1996, pp. 1415-1418.

[5] S. Trabelsi, A. W. Kraszewski, and S. O. Nelson, "Simultaneous determination of density and water content by microwave sensors," Electron. Lett., vol. 33, no. 10, pp. 874-876, 1997.

[6] - "Determining physical properties of grain by microwave permittivity measurements," Trans. ASAE, vol. 42, no. 2, pp. 531-536, 1999.

[7] - "Unified calibration method for nondestructive dielectric sensing of moisture content in granular materials," Electron. Lett., vol. 35, no. 16, pp. 1346-1347, 1999.

[8] _ "New density-independent calibration function for microwave sensing of moisture content in particulate materials," IEEE Trans. Instrum. Meas., vol. 47, pp. 613-622, June 1998.

[9] S. Trabelsi and S. O. Nelson, "Density-independent functions for on-line microwave moisture meters: A general discussion," Meas. Sci. Technol., vol. 9, no. 4, pp. 570-578, 1998.

[10] ASAE Standards 1999:, Moisture Measurement-Unground Grain and Seeds. St. Joseph, MI: Amer. Soc. Agric. Engrs., 1999, ASAE S352.2.

[11] S. O. Nelson, "Review of factors influencing the dielectric properties of cereal grains," Cereal Chem., vol. 58, no. 6, pp. 487-492, 1981.

[12] A. von Hippel, Dielectric Materials and Applications. New York: Wiley, 1954.

[13] E. Nyfors and P. Vainikainen, Industrial Microwave Sensors. Norwood, MA: Artech House, 1989.

[14] A. W. Kraszewski, S. Trabelsi, and S. O. Nelson, "Wheat permittivity measurements in free space," J. Microw. Power Electromagn. Energy, vol. 31, no. 3, pp. 135-141, 1996.

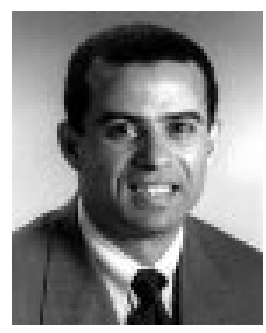

Samir Trabelsi (M'89-SM'98) was born in Sfax, Tunisia, in 1960. He received the Lic. Phys. and Maitrise de Physique degrees from l'Universite Paul Sabatier, Toulouse, France, in 1985 and 1987, respectively, and the Diplome d'Etudes Approfondies and the Ph.D. degree in electronics from the Institut National Polytechnique de Toulouse in 1988 and 1993, respectively.

Since 1994, he has been a Visiting Scientist (Electronics Engineer) in the Dielectrics Group, Quality Assessment Research Unit at the Richard B. Russell Agricultural Research Center, U.S. Department of Agriculture (USDA), Agricultural Research Service, Athens, GA. His current research involves development of principles and calibration methods for microwave-sensing devices for on-line nondestructive determination of density and moisture content in particulate and granular materials and measurements and modeling of dielectric properties of heterogeneous substances at microwave frequencies. He has published more than 50 technical papers and reports on these subjects and holds two patents.

Dr. Trabelsi is the co-inventor of a portable microwave moisture meter, for which the First Prize was awarded in the 15th Regional Competition of Innovation, Southwest Area, France, in 1995. He is listed in Marquis Who's Who in Science and Engineering. He is a member of Sigma Xi, the Scientific Research Society, and a member of the American Society of Agricultural Engineers. 


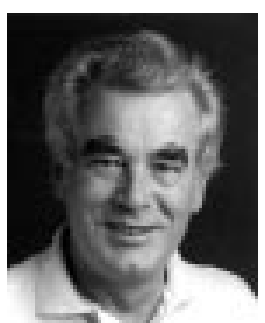

Andrzej W. Kraszewski (M'89-SM'91-F'96) was born in Poznan, Poland, on April 22, 1933. He received the B.Sc. and M.Sc. degrees in electrical engineering from the Technical University of Warsaw, Warsaw, Poland in 1954 and 1958, respectively, and the D.Sc. degree in technical sciences from the Polish Academy of Sciences (PAN), Warsaw, in 1973.

In 1953, he joined the Telecommunication Institute (PIT), Warsaw, where he did research and development of microwave systems and components. In 1963, he joined UNIPAN Scientific Instruments, a subsidiary of the Polish Academy of Sciences, Warsaw, as Head of the Microwave Laboratory. In 1972, he became the Manager of the Microwave Department of WILMER Instruments and Measurements, a subsidiary of the Polish Academy of Sciences, Warsaw, where he co-developed microwave instrument for moisture content measurement and control. Beginning in November 1980, he was a Visiting Professor at the University of Ottawa, Ottawa, ON, Canada, where he did research on RF and microwave dosimetry. In January 1987, he joined the Richard B. Russell Agricultural Research Center, U.S. Department of Agriculture (USDA), Athens, GA, where he is involved in research on plan structure and composition and quality assessment of agricultural products using electromagnetic fields. He is the author of several books on microwave theory and techniques, has published close to 300 technical papers on the subject, and holds more than 20 patents.

Dr. Kraszewski is a member of Sigma Xi, the International Microwave Power Institute, the Materials Research Society, the New York Academy of Sciences, and the Polish Electricians Association (SEP). He received several professional awards, among them the State Prize in Science in Poland in 1980.

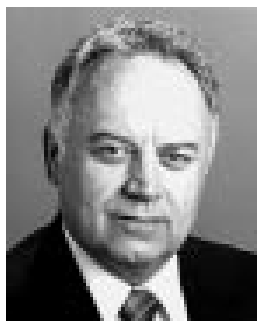

Stuart O. Nelson (SM'72-F'98) was born in Stanton County, NE, in 1927. He received the B.S. and M.S. degrees in agricultural engineering and the M.A. degree in physics from the University of Nebraska, Lincoln, in 1950, 1952, and 1954, respectively, and the $\mathrm{Ph} . \mathrm{D}$. degree in engineering from Iowa State University, Ames, in 1972.

From 1954 to 1976, he was a Research Engineer with the U.S. Department of Agriculture (USDA), Agricultural Research Service, Lincoln, NE, and Research Leader for Electromagnetic Radiation Investigations nationally. $\mathrm{He}$ was Professor of Agricultural Engineering and Graduate Faculty Fellow at the University of Nebraska. In 1976, he transferred his laboratory to the USDA's Richard B. Russell Agricultural Research Center, Athens, GA, where he is an Adjunct Professor and a member of the Graduate Faculty at the University of Georgia, Athens. His research interests include the use of radio-frequency and microwave dielectric heating for seed treatment, stored-grain insect control, and agricultural product conditioning; studies of the dielectric properties of grain, seed, insects, coal, and minerals; methods of dielectric properties measurement; dielectric properties and density relationships in granular and pulverized materials; and moisture measurement through sensing dielectric properties of agricultural products. These studies have been documented in more than 400 publications.

Dr. Nelson is a member of ASAE, IMPI, AMPERE, AAAS, NSPE, CAST, OPEDA, Sigma Tau, Sigma Xi, Gamma Sigma Delta, and Tau Beta Pi. He is a Fellow of ASAE and IMPI. Honors include the IMPI Decade Award, NSPE Founder's Gold Medal as the 1985 Federal Engineer of the Year, USDA Superior Service Award, Professional Achievement Citation in Engineering from Iowa State University, the OPEDA Professional-of-the-Year Award, and election to the National Academy of Engineering in 1990. He was awarded an honorary doctor of science degree by the University of Nebraska, Lincoln, in 1989 and inducted into the University of Nebraska Biological Systems Engineering Hall of Fame in 1999. The Georgia Engineering Foundation awarded him the 1999 Medal of Honor, and he received the McCormick-Case Gold Medal Award from ASAE in 2000 for exceptional and meritorious engineering achievement in agriculture. 